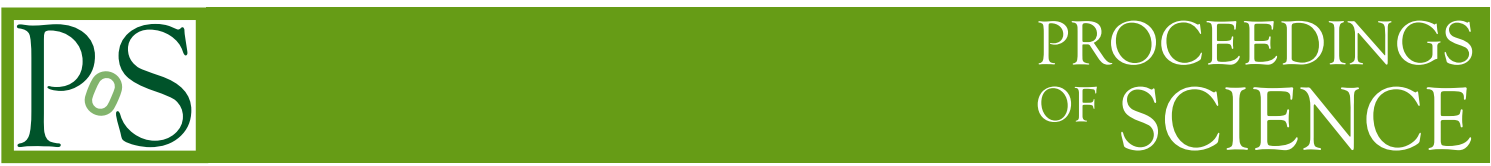

\title{
3D sensors for tracking detectors: present and future applications
}

\author{
Claudia Gemme* \\ Istituto di Fisica Nucleare \\ Sezione di Genova \\ E-mail: Claudia.Gemmedcern.ch
}

Pixel detectors with cylindrical electrodes that penetrate the silicon substrate (so called 3D detectors) offer advantages over the standard planar sensors in terms of radiation hardness, since the electrode distance is decoupled from the bulk thickness. Recently, the 3D technology has reached a mature level that has culminated in the production of the sensors for the ATLAS Insertable B-Layer (IBL), the first upgrade for the innermost tracking detector. Based on this positive experience, the $3 \mathrm{D}$ technology is envisaged for future detectors. This paper reviews the status of the IBL production as well as the 3D usage foreseen in future detectors, namely the tracking detectors for forward detectors of the ATLAS and CMS upgrades and also for the future trackers foreseen for 2022-2023 upgrades.

22nd International Workshop on Vertex Detectors,

15-20 September 2013

Lake Starnberg, Germany

\footnotetext{
* Speaker.
} 


\section{Introduction}

The unique structure of 3D silicon sensors [1], featuring columnar electrodes penetrating all the way through the substrate, enables the active thickness to be decouped from the inter-electrode spacing, offering important advantages in terms of low operation voltage and high charge collection efficiency in irradiated devices [2] with respect to standard silicon planar sensors. In the past few years, very important progress has been made in the devolpment of 3D sensors, passing from the earlier RD phase with performance demonstration of few prototypes (1997-2006) to more systematic studies (2007-2010) and finally to an industrialization/qualification phase (2011/2012), which has led to the production of 3D sensors to provide $25 \%$ of the Insertable B-Layer (IBL) [3] in the ATLAS [4] pixel upgrade of 2014.

Given their intrinsic radiation hardness, 3D sensors are very attractive for the future upgrades at the LHC Phase-II, although several improvements will be needed to face an even harsher environment, with increased radiation fluence, very high charged particles density and the request for a reduced material budget .

In the following Section the experience of the 3D sensor production for IBL is reviewed, reporting measurements at the wafer and module levels, aiming to emphasize the learned lessons from the very first experience of this technology assembled as a real detector. In Section 3 and 4 the status of the prototipization for an ATLAS Forward Detector and in CMS is revisited, respectively.

\section{IBL}

The IBL sensors and front-end electronics will be located at an average radius of $\approx 34 \mathrm{~mm}$ from the beam axis. Such a small radius implies severe requirements for the IBL modules [5]. For an integrated luminosity up to $\approx 550 \mathrm{fb}^{-1}$, including safety factors, the IBL needs to withstand 250 Mrad of ionizing dose and $5 \times 10^{15} \mathrm{n}_{e q} / \mathrm{cm}^{2}$ of non-ionizing dose, that corresponds to a factor 5 larger radiation hardness with respect to the current Pixel Detector requirement.

The IBL will consist of 14 pixel staves surrounding the beam-pipe. Each carbon-fibre stave carries and provides cooling to 32 FE-I4 read-out chips [6], each one organized in a array of $50 \times$ $250 \mu \mathrm{m}^{2}$ pixels, bump-bonded to silicon sensors. Two types of sensors will be used: planar $\mathrm{n}-$ in-n sensors, similar to the present Pixel Detector [7] in the center of the staves and 3D silicon sensors at the edges. In case of planar sensors, one sensor tile carries two FE-I4 chips, in case of 3D sensors, one sensor carries one FE-I4 chip. The staves are inclined by $14^{\circ}$ with respect to the radial direction in order to achieve overlap of active area between staves and to compensate for (a) the Lorentz angle of drifting charges in the $2 \mathrm{~T}$ magnetic field in case of planar sensors or (b) the effect of partial column inefficiency with perpendicular tracks in case of 3D sensors. There is no shingling of sensors along $z$ due to the lack of radial space. Modules are glued on the stave with a physical gap of $200 \mu \mathrm{m}$ for planar two-chips modules and $100 \mu \mathrm{m}$ in case of single-chip 3D modules. The $643 \mathrm{~mm}$ long active area of a stave corresponds to a pseudo-rapidity coverage of $|\eta|<3$.

With 112 modules installed, the IBL represents the first 3D large scale tracking detector. 


\subsection{D sensors}

In the 3D sensor design the measuring and biasing electrodes are etched through the $\mathrm{p}+$ silicon bulk by Deep Reactive Ion Etching (DRIE), as shown in Figure 1. The sensor is depleted between $\mathrm{n}^{+}$pixel electrodes and $\mathrm{p}^{+}$biasing electrodes. This biasing scheme makes the sensor depletion independent of the sensor thickness and allows for high field strength and good charge collection after irradiation at moderate bias voltages. For the IBL, a design with two $\mathrm{n}^{+}$electrodes per pixel, surrounded by six $\mathrm{p}^{+}$biasing electrodes, has been chosen to optimize the maximum collected charge and minimum electronics noise due to detector capacitance [2]. The sensors are $230 \mu \mathrm{m}$ thick and are manufactured in double-sided processes by $\mathrm{CNM}^{1}$ and $\mathrm{FBK}^{2}$. The sensors manufactured by the two foundries share the same top metal layout for identical bump-bonding connection to the electronics. Both 3D designs by CNM and FBK feature the edge pixel of standard $250 \mu \mathrm{m}$ length and an inactive edge of $200 \mu \mathrm{m}$ width. The main difference between the two sensor versions regards the column depth: as shown in Fig. 1, in CNM sensors columns do not traverse the substrate but stop at few microns from the surface of the opposite side, whereas FBK sensors [8] have traversing columns. Another difference concerns the isolation implantation between the $\mathrm{n}^{+}$columns at the surface: p-stops are implanted on the front side of CNM sensors while FBK sensors use p-spray implantations on both sides. The slim edge guard ring design in CNM sensors is made using the combination of $\mathrm{a} \mathrm{n}^{+}$3D guard ring that is grounded, and fences that are at the bias voltage from the ohmic side. In FBK sensors, the slim edge fence consists of several rows of ohmic columns that effectively stop the lateral depletion region from reaching the cut line, thus significantly increasing the shielding of the active area from edge effects [9].
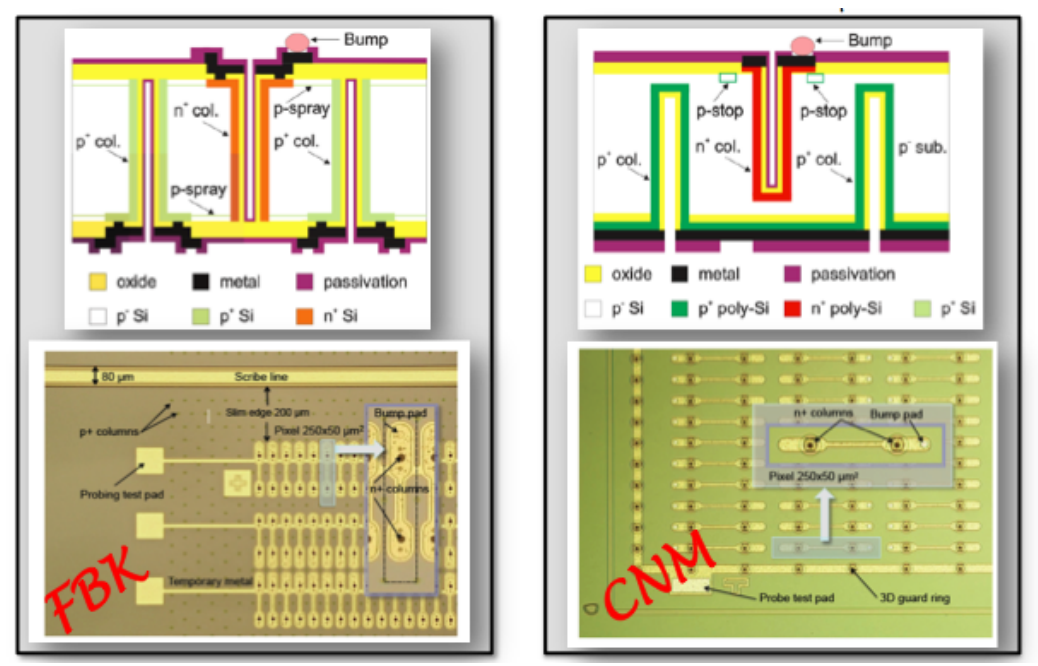

Figure 1: Etched columns from FBK and CNM (top), FBK temporary metal (bottom left), CNM guard ring (bottom right).

As extensively reported in Ref. [5], both 3D sensors types fullfill the IBL tight requirements, namely: hit efficiency $>97 \%$ at the end of the detector lifetime, minimal inactive edge (typically

\footnotetext{
${ }^{1}$ Centro Nacional de Microelectronica, Barcelona, Spain http://www.cnm.es/

${ }^{2}$ Fondazione Bruno Kessler, Trento, Italy, http://www.fbk.eu/
} 
$200 \mu \mathrm{m}$ ), bias voltage $\leq 1000 \mathrm{~V}$ and power dissipation below $200 \mathrm{~mW} / \mathrm{cm}^{2}$ for a sensor temperature of $-15^{\circ} \mathrm{C}$.

\subsection{D sensor and modules production}

Once qualified by CNM and FBK, selected wafes are sent to $\mathrm{IZM}^{3}$ to be assembled to the FE-I4 read-out chip using SnAg solder bump-bonding. Due to the large chip size and its reduced thickness, $\approx 150 \mu \mathrm{m}$, a handling wafer process has been devised: the chip is thinned and a glass handling wafer is bonded to the thinned chip wafer using a photo-sensitive adhesive. After the flip-chip and reflow process the glass is removed by means of laser exposure of the adhesive. In Bonn and Genova laboratories modules assembly is completed gluing on the back of the sensor a flex circuit with passive components that will be used to establish the connection via wire-bondings to the services once the modules will be loaded on the final mechanical supports.

The main measurements used for sensor production quality assurance (QA) are I-V curves that are performed on the wafer and once fully assembled into modules. At the wafer scale I$\mathrm{V}$ measurements are made on each sensor using probe stations, exploiting a removable temporary metal (FBK) allowing a measurement of I-V at the column level or measuring the guard ring current only (CNM). Working 3D sensors are then mainly required to have breakdown voltage $\left|V_{b k}\right|>25 \mathrm{~V}$ and leakage current $<2 \mu \mathrm{A}$ at $20 \mathrm{~V}$. Wafers are then selected for bump-bonding if they have at least 3 out of eight working sensors. As the CNM measurement is more rough than the FBK one, the yield of selected wafers is higher for CNM $(\approx 80 \%)$ than for FBK $(\approx 50 \%)$ as well as the number of selected tiles (57\% vs $25 \%$ ). However, since the very first tests of assembled modules, a much better correlation between the breakdown voltage at the wafer and module level has been found for FBK than for CNM, as shown in Fig. 2. To avoid flip-chipping and assembly of poor quality sensors, a re-measurement campaign of the diced sensors not yet used has been put in place. Having the sensors the under bump metallisation, all the pixels could be contacted. The measurement has confirmed a yield of $65 \%$ when considering the full sensor and not the guard ring only, thus making the overall yield for the two producers rather comparable.
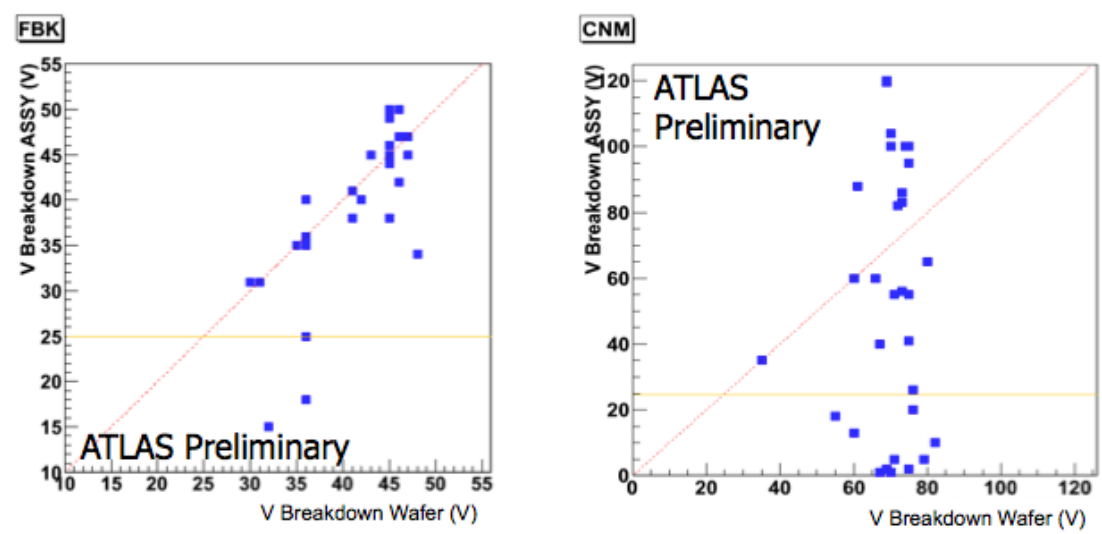

Figure 2: Correlation of breakdown voltage at the wafer and module level for FBK and CNM.

\footnotetext{
${ }^{3}$ Fraunhofer IZM, Berlin, Germany, http://www.izm.fraunhofer.de/
} 
Typical values for the electronics noise are $140 e^{-}$(FBK) and $120 e^{-}(\mathrm{CNM})$ in operating conditions, $-10{ }^{0} \mathrm{C}$ temperature and $20 \mathrm{~V}$ bias $\left(\mathrm{V}_{b}\right)$. The noise increases if the voltage bias decreases, and in particular if there is no bias applied. This is a good method to tag disconnected bumps, as if the pixel electronics cell is not connected to the sensor the noise is lower and it does not depend on the bias conditions. Figure 3 shows the difference for each pixel between the noise at $\mathrm{V}_{b}=20 \mathrm{~V}$ and $\mathrm{V}_{b}$ off. A cut of $20 e^{-}$is very efficient at room temperature, while at low temperature detects $10 \%$ fakes for FBK sensors. Therefore during the module qualification at cold temperature also a source scan is used to count the disconnected bumps.

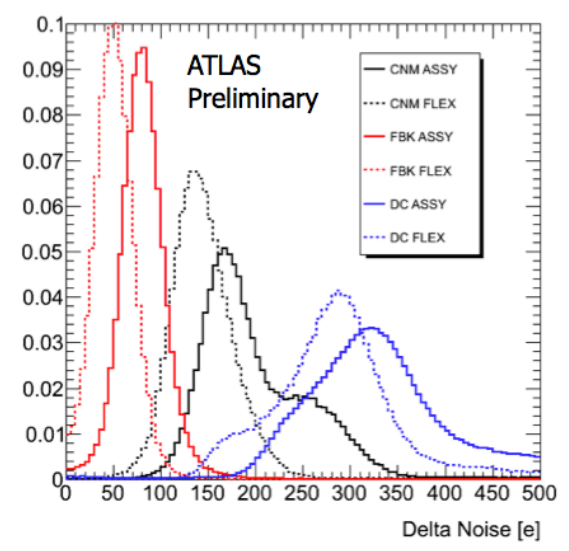

Figure 3: Pixel-by-pixel difference between noise at bias voltage $\mathrm{V}_{b}=20 \mathrm{~V}$ and noise with $\mathrm{V}_{b}$ off for planar (blue), FBK (red), CNM (black) devices at room (solide line) or cold (dotted line) temperature.

The different design of the edge region makes the charge collection of the peripheral pixels different for the two vendors. Figure 4 shows the occupancy of the individual pixel during an ${ }^{241} \mathrm{Am}$ scan. The FBK edges are partially active: hit occupancy of edge pixels (by rows and columns) is larger than for the internal pixels. For CNM sensors, the guard ring collects the charge outside the pixels area: the hit occupancy of peripheral pixels is therefore the same as the internal ones.
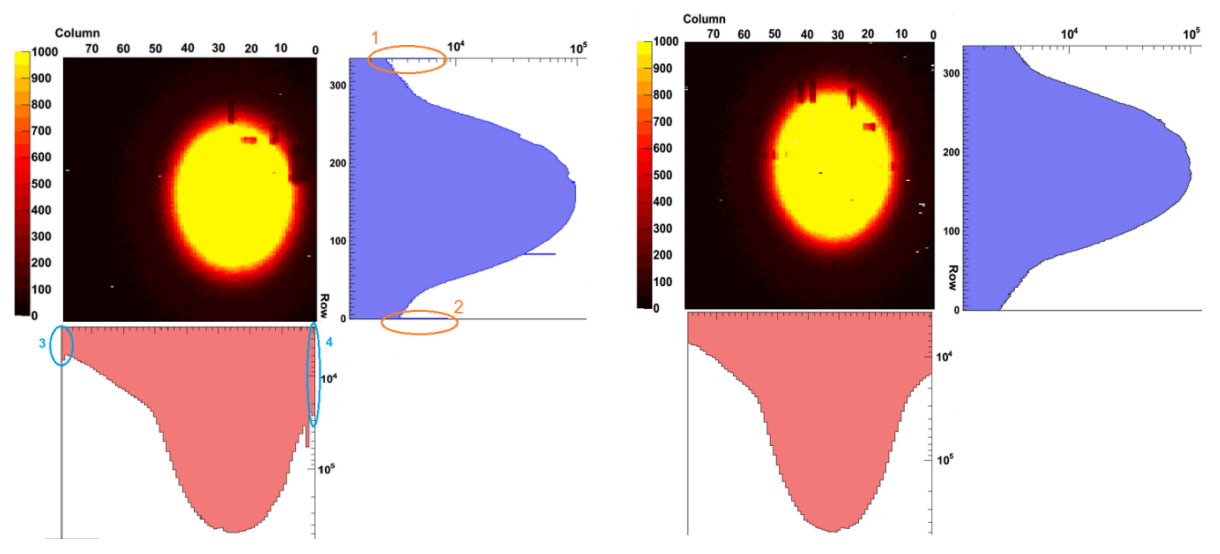

Figure 4: Occupancy maps and projections on rows and columns for FBK (left) and CNM (right) devices (logarithmic scale) of a ${ }^{241} \mathrm{Am}$ source scan. 
The inter-pixel cross-talk is measured by injecting the maximum charge $\left(\approx 50 \mathrm{k} e^{-}\right)$in two neighbouring pixels and counting the number of hits above threshold in the central one. The test is run routinely at $3 \mathrm{k} e^{-}$threshold. As usually no cross-talk hits are detected, the cross-talk is lower than 3\%. However for some CNM devices some cross-talk hits are measured, although to a level not worrisome for data-taking in operation conditions. Cross-talk hits dependence on the injected charge at different threshold conditions are shown for one device in Figure 5.

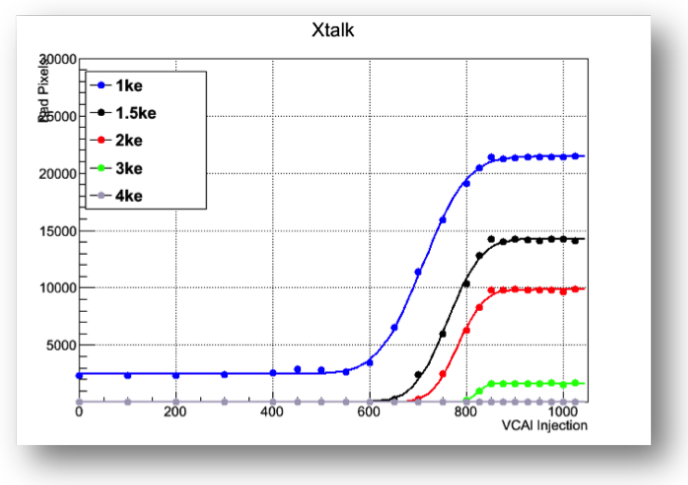

Figure 5: Number of pixels that detect cross-talk hits as a function of the injected charge. 1 VCal DAC corresponds to $\approx 50 e^{-}$. The cross-talk increases as the threshold is lower.

Overall the yield of the 3D sensors at the module level is $\approx 70 \%$ for FBK and $\approx 60 \%$ for CNM. The failures are mainly due to bump-bonding issues in the first 3 batches [10]. Other sources of failures, affecting all the production batches, are mechanical damages in shipping or assembly, low breakdown voltage or high leakage currents, mainly for CNM as explained before, electrical problems, mainly related to the FEI 4 regulators. Figure 6 shows the failure rates for FBK and CNM modules in the flip-chip batches.
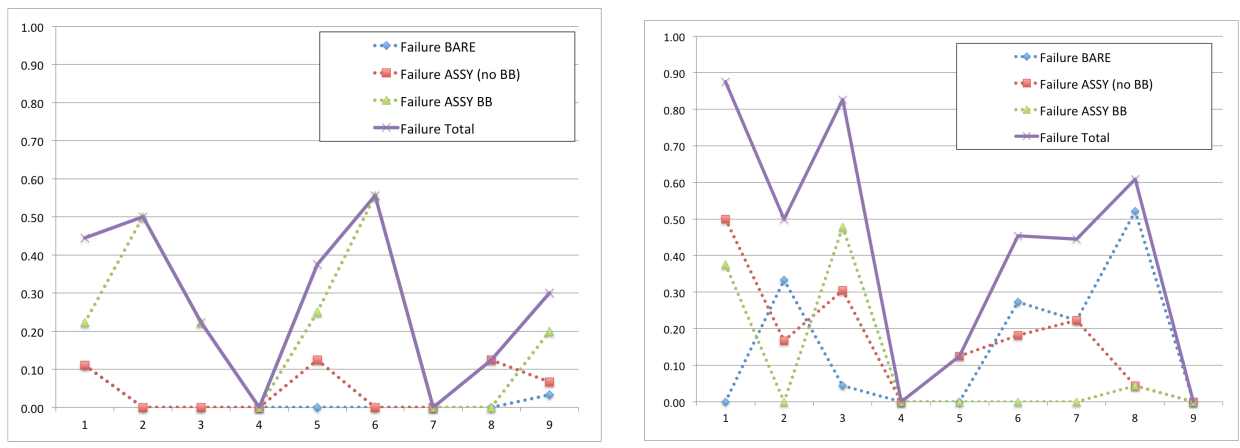

Figure 6: Failure rate for FBK and CNM for the delivered modules in IZM batches. Failures are shown before assembly (BARE), after assembly due to bump-bonding (ASSY BB) or to other reasons (ASSY no $\mathrm{BB})$.

Once modules are loaded on the stave, a full qualification takes place, considering also system aspects. Overall 3D sensors are very stable and behave as expected, also at the lowest operational threshold of $1500 e^{-}$. 


\section{AFP experience}

ATLAS intends to install a Forward Physics detector (AFP) in order to identify diffracted protons at $\approx 210 \mathrm{~m}$ from the interaction point in 2018 [11, 12]. The current AFP design foresees a high resolution pixel silicon tracking system combined with a timing detector for the removal of pile-up protons. The AFP tracker unit will consist of an array of six pixel sensors placed at 2-3 $\mathrm{mm}$ from the Large Hadron Collider (LHC) proton beam. The proximity to the beam is essential for the AFP physics program as it directly increases the sensitivity of the experiment. Thus, there are two critical requirements for the AFP pixel detector: first, the active area of the detector has to be as close as possible to the LHC beam, which means that the dead region of the sensor has to be minimized. Second, the device has to be able to cope with a very inhomogeneous radiation distribution. Preliminary estimations indicate that the side of the sensors close to the beam will have to sustain a fluence equivalent to $\approx 5 \times 10^{15} \mathrm{n}_{e q} / \mathrm{cm}^{2}$ while the opposite side is expected to receive several orders of magnitude less radiation.

AFP modules [13] will be very similar to those used in IBL, consisting in FEI4 read-out chip assembled with $3 \mathrm{D}$ sensors. However, the IBL sensor design has a large $(\approx 1 \mathrm{~mm})$ dead region in the side opposite to the wirebonds, not critical as the sensors overlap in the $r-\phi$ direction. This region has to be reduced for the AFP modules as it will be the closest to the beam. Few techniques to adapt the 3D IBL sensors for AFP has been attempted. Recently, two CNM and two FBK devices have been reduced to a slim edge of $\approx 100 \mu \mathrm{m}$, see Fig. 7, and preliminary test beam studies show an hit efficiency of $\approx 98.3 \%$.
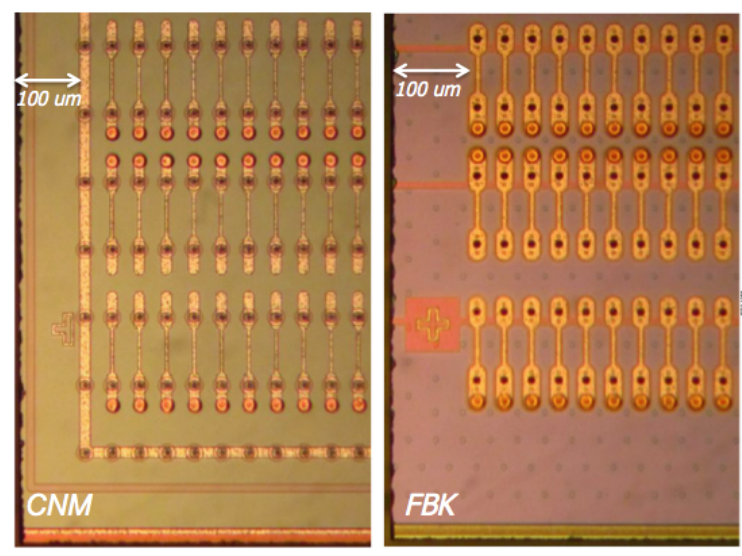

Figure 7: Reduced edges for FBK and CNM sensors.

The other critical aspect for the AFP sensors is the inhomogeneous irradiation. This means that the less irradiated area of the sensor have to sustain high bias voltages keeping the leakage current at moderate values. Few FEI4 CNM devices have been irradiated non-uniformly at the IRRAD1 facility at CERN-PS. I-V curve of one of those is shown in Fig. 8. Besides the good leakage current, the device has also tested with a $120 \mathrm{GeV}$ pions beam at the CERN SPS H6. Once the dead and noisy pixel cells (due to front-end issues) are masked, the hit efficiency is $\approx 98 \%$ in the high-irradiated side. 


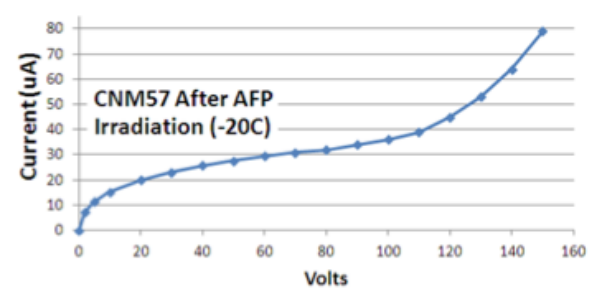

Figure 8: Measurement of the leakage current as a function of the bias voltage for one not-uniformly irradiated device. The maximum dose for this module was $4.0 \times 10^{15} \mathrm{n}_{e q} / \mathrm{cm}^{2}$.

\section{CMS experience}

Similar to ATLAS, the CMS [14] collaboration is considering the 3D detectors for two projects: as internal layers of the vertex detector for the LHC Phase-II upgrade and for the PPS detector [15], a near-beam proton spectrometer to be installed at $\approx 220 \mathrm{~m}$ from the interaction point.

In the following some results of FBK sensors are summarized; details can be found in Ref. [16, 17]. Three different pixel configurations were designed and manufactured, each of them compatible with the existing CMS pixel readout chip (ROC) type PSI46v2 [18], which has an array of 80 rows $\times 52$ columns of $100 \mu \mathrm{m} \times 150 \mu \mathrm{m}$ readout pixels. The different pixel configurations differs in the number of n-type electrodes per cell and the resulting inter-electrode distances in the $1 \mathrm{E}, 2 \mathrm{E}$, and $4 \mathrm{E}$ configurations are $90 \mu \mathrm{m}, 62.5 \mu \mathrm{m}$ and $45 \mu \mathrm{m}$, respectively. FBK sensors, $200 \mu \mathrm{m}$ thick, were diced and bump bonded to the readout chip at SELEX Sistemi Integraty (Italy) using Indium bumps. Laboratory tests were performed in Torino and Purdue laboratories. Most of the devices performed well, showing leakage currents of less than $300 \mathrm{nA}$ and breakdown voltages between 20 and $60 \mathrm{~V}$. The relatively low breakdown voltage was understood and attributed to the too high pspray implanted dose, but it does not affect the charge collection efficiency as 3D devices are fully depleted at around $10 \mathrm{~V}$. Noise decreases as reverse bias increases as expected from $\mathrm{C}-\mathrm{V}$ behaviour and it increases with electrode number, as shown in Fig. 9. Average sensor noise at full depletion is $230 e^{-}$for $1 \mathrm{E}$ sensors, $280 e^{-}$for $2 \mathrm{E}$ sensors, and $330 e^{-}$for $4 \mathrm{E}$ sensors.

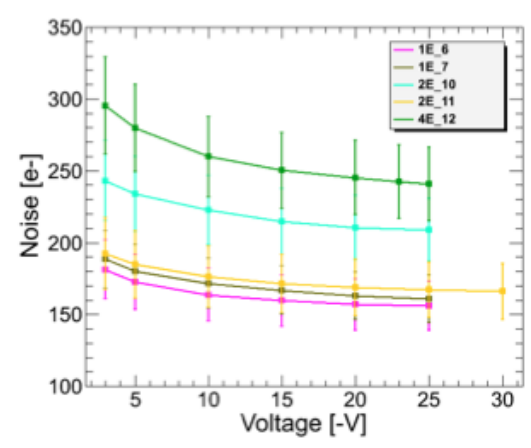

Figure 9: Measurement of the noise versus the reverse bias for FBK devices with different electrodes configurations. 
Charge collection has been studied with a ${ }^{90} \mathrm{Sr}$ radioactive source and its most probable value (MPV) is consistent with expectations. Sensor charge collection increases for increasing bias voltages until it reaches a plateau. Saturation begins at $10 \mathrm{~V}$ reverse bias for most of the sensors, regardless of their electrode configuration, in good agreement with the full depletion voltage extracted from the $\mathrm{C}-\mathrm{V}$ curves.

Sensor performances have been studied in beam tests at the Meson Test facility of Fermilab. The collected charge is what is expected in the sensor thickness only if the track hits the central part of the pixel, otherwise it is lower due to charge sharing and readout chip threshold setting (6 $\left.\mathrm{k} e^{-}\right)$. The efficiency increases with the bias voltage and saturates at $20 \mathrm{~V}$, where the detector is completly depleted. Fig. 10 shows that the efficiency increases from a value of $\approx 97.5 \%$ when the sensor is orthogonal to the beam $\left(0^{\circ} \mathrm{C}\right)$, the electrodes being inactive volumes for track impinging orthogonal to the detector, to $\approx 98.5 \%$ at an angle of $20^{\circ} \mathrm{C}$. The spatial resolution for two-pixel clusters was measured to be $\approx 11 \mu \mathrm{m}(\approx 13 \mu \mathrm{m})$ in the short (long) dimension at $\left(20^{\circ} \mathrm{C}\right)$ and 20 $\mathrm{V}$ bias. A few detectors have been irradiated at Los Alamos National Laboratory with $800 \mathrm{MeV}$ protons up to fluences of $3.5 \times 10^{15} \mathrm{n}_{e q} / \mathrm{cm}^{2}$ and then their properties have been measured in the beam test. The breakdown voltage did not increase significantly after irradiation due to the too high p-spray dose of this batch. All sensors show lower collected charge after irradiation; the effect is more evident in $1 \mathrm{E}$ sensors ( $35 \%$ loss in $1 \mathrm{E}$ and $8 \%$ in $2 \mathrm{E}$ after $0.7 \times 10^{15} \mathrm{n}_{e q} / \mathrm{cm}^{2}$ ) due to the greater electrode distance and the consequent higher trapping probability. The variation in both charge collection and efficiency is higher for the sensors which underwent higher fluence.

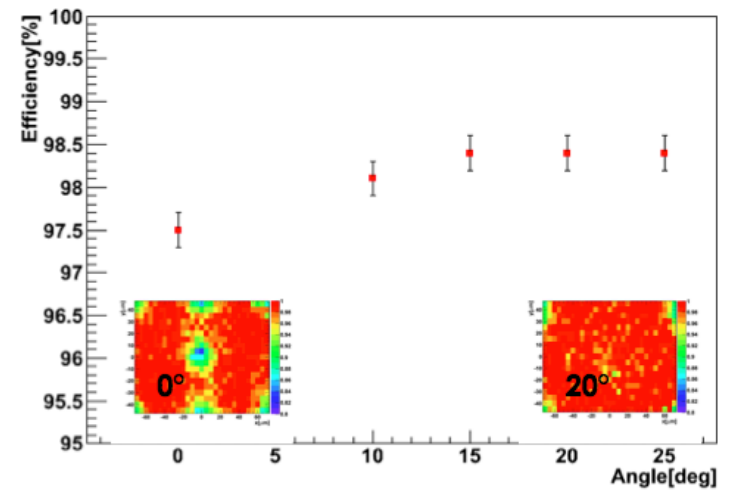

Figure 10: Efficiency versus the angle between the detector and the beam, measured at $20 \mathrm{~V}$ for an unirradiated $1 \mathrm{E}$ sensor. In the boxes the pixel map of the efficiency at $0^{\circ} \mathrm{C}$ (left) and $20^{\circ} \mathrm{C}$ (right).

\section{Outlook}

The successful production of IBL modules, being the 3D first large scale detector, opens the way to the future use of 3D detectors in high energy physics, primarily in the harsh conditions of HL-LHC. With higher radiation dose $\left(2 \times 10^{16} \mathrm{n}_{e q} / \mathrm{cm}^{2}\right)$ and higher pile-up (140 events/bunch crossing), smaller pixel size, lower threshold, lower material budget will be needed to maintain equivalent tracking performances. On the other hand, optimization of the production process to have a higher yield is certainly welcome as well as more controlled selection at wafer level to 
reduce costs. In parallel very interesting developments are on-going in view of future applications: a report on AFP modules that will suffer inhomogenous fluence and will need slim edges has been presented and CMS measurements of 3D sensors.

Many thanks to D. Bortoletto, C. Da Via, S. Grinstein, M. Obertino and A. Solano for providing material and helpful discussions while preparing the talk for the Vertex 2013 Conference.

\section{References}

[1] S. Parker, C. J. Kenney, J. Segal, "3D: A proposed new architecture for solid-state radiation detectors", Nucl. Instrum. Methods A, vol. 395, no. 3, pp. 328-343, 1997.

[2] C. Da Via et al., "3D active edge silicon sensors with different electrode configurations: Radiation hardness and noise performance", Nucl. Instrum. Methods A, vol. 604, pp. 505-511, 2009.

[3] The ATLAS Collaboration, "Insertable B-layer, Technical Design Report", CERN-LHCC-2010-013.

[4] The ATLAS Collaboration, "The ATLAS Experiment at the CERN Large Hadron Collider", 2008 JINST 3 S08003.

[5] The ATLAS IBL Collaboration, "Prototype ATLAS IBL Modules using the FEI4A Front End Readout Chip", 2012 JINST 7 P11010.

[6] M. Garcia-Sciveres et al., "The FE-I4 pixel readout integrated circuit", Nucl. Instrum. Methods in Physics Research A, vol. 636, pp. 155-159, 2011.

[7] G Aad et al., "ATLAS Pixel Detector electronics and sensors", 2008 JINST 3 P07007.

[8] G.-F. Della Betta et al., "Development of modified 3D detectors at FBK", Nuclear Science Symposium Conference Record (NSS/MIC), 2010 IEEE, Nov. 2010, pp. 382-387.

[9] M. Povoli et al., "Slim edges in double-sided silicon 3D detectors", 2012 JINST 7 C01015.

[10] F.Huegging, these proceedings.

[11] The ATLAS Collaboration, Letter of Intent for the Phase-I Upgrade of the ATLAS Experiment, CERN-LHCC-2011-012, 2011

[12] L. Adamczyk et al., "AFP: A proposal to install proton detectors at $220 \mathrm{~m}$ around ATLAS to complement the ATLAS high luminosity physics program", ATL-COM-LUM-2011-006, CERN (2011), AFP technical proposal.

[13] S.Grinstein et al., "Beam teststudies of 3D pixel sensors irradiated non-uniformly for the ATLAS forward physics detector", Nucl. Instrum. Methods A, vol. 730, pp. 28-32, 2013.

[14] The CMS Collaboration, "The CMS Experiment at the CERN Large Hadron Collider", 2008 JINST 3 S08004.

[15] M. G. Albrow, "Precision proton spectrometers for CMS", arXiv:1310.4529, EDSBLOIS-2013-42; M.G.Albrow et al., "FP420 Report", 2009 JINST 4 T10001.

[16] E. Alagoz et el., "Simulation and laboratory test results of 3D CMS pixel detectors for HL-LHC", 2012 JINST 7 P08023.

[17] M.Obertino et al., "Performance of CMS 3D silicon pixel detectors before and after irradiation", Nucl. Instrum. Methods A, vol. 730, pp. 33-37, 2013.

[18] H.C. Kastli et al., "Design and performance of the CMS pixel detector readout chip", Nucl. Instrum. Methods A, vol. 565, pp. 188-194, 2006. 